\title{
A Hollow-Waveguide Gas Correlation Radiometer for ultra-precise column measurements of formaldehyde on Mars
}

\author{
Emily LWilson, ${ }^{1}$ Marc Neveu, ${ }^{2,3}$ Haris Riris, ${ }^{1}$ Elena M Georgieva, ${ }^{4}$ and William \\ S Heaps ${ }^{1}$ \\ ${ }^{1}$ NASA Goddard Space Flight Center, Laser and Electro-Optics Branch, \\ Greenbelt, MD, 20771, USA \\ ${ }^{2}$ NASA Academy, Goddard Space Flight Center, Greenbelt, MD 20771, USA \\ ${ }^{3}$ Institut Superieur de l'Aeronautique et de l'Espace, Toulouse, France \\ ${ }^{4}$ Goddard Earth Sciences and Technology Center, Baltimore, MD, 21228, USA
}

E-mail: Emily.L.Wilson@,nasa.gov

\begin{abstract}
We present preliminary results in the development of a miniaturized gas correlation radiometer that implements a hollow-core optical fiber (hollow waveguide) gas correlation cell. The substantial reduction in mass and volume of the gas correlation cell makes this technology appropriate for an orbital mission - capable of pinpointing sources of trace gases in the Martian atmosphere. Here we demonstrate a formaldehyde $\left(\mathrm{H}_{2} \mathrm{CO}\right)$ sensor and report a detection limit equivalent to $\sim 30 \mathrm{ppb}$ in the Martian atmosphere. The relative simplicity of the technique allows it to be expanded to measure a range of atmospheric trace gases of interest on Mars such as methane $\left(\mathrm{CH}_{4}\right)$, water vapour $\left(\mathrm{H}_{2} \mathrm{O}\right)$, deuterated water vapour $(\mathrm{HDO})$, and methanol $\left(\mathrm{CH}_{3} \mathrm{OH}\right)$. Performance of a formaldehyde instrument in a Mars orbit has been simulated assuming a 3 meter long, 1000 micron inner diameter hollow-core fiber gas correlation cell, a 92.8 degree sunsynchronous orbit from $400 \mathrm{~km}$ with a horizontal sampling scale of $10 \mathrm{~km} \times 10 \mathrm{~km}$. Initial results indicate that for one second of averaging, a detection limit of $1 \mathrm{ppb}$ is possible.
\end{abstract}

Keywords: hollow waveguide, hollow optical fiber, gas correlation radiometer, passive, remote sensing, Mars, methane, formaldehyde, water vapor, isotopic ratios. 


\section{Introduction}

The Martian atmosphere presents many exciting opportunities to investigate meteorology, atmospheric origin, chemical stability, and atmospheric dynamics. One of the most interesting questions about Mars is the source of trace amounts of methane $\left(\mathrm{CH}_{4}\right)$ in the atmosphere - and whether these originate from geological sources such as gas seeps, active volcanism and serpentization reactions (associated with ancient olivine-bearing crustal rocks), or from the presence of extant life [1-3].

The source of methane can be revealed through measurements of trace disequilibrium gases that are likely to be found together with methane. For example, trace gases such as ethane $\left(\mathrm{C}_{2} \mathrm{H}_{6}\right)$, nitrous oxide $\left(\mathrm{N}_{2} \mathrm{O}\right)$, and hydrogen sulfide $\left(\mathrm{H}_{2} \mathrm{~S}\right)$ found with methane would indicate a biogenic source. On Earth, methane and ethane are produced in similar abundance in cold deepsea sediments [4]. With the exception of anthropogenic sources, nitrous oxide is predominantly a product of microbial action in wet tropical forests. Hydrogen sulfide is produced by bacterial activity in oxygen-depleted environments (e.g. swamps). Sulfur dioxide $\left(\mathrm{SO}_{2}\right)$ found with methane would point to a geologic origin. Sulfur dioxide has been found in shergottite samples as well as terrestrial magmas [5].

The occurrence of formaldehyde $\left(\mathrm{CH}_{2} \mathrm{O}\right)$ with methane is of particular interest as an indicator of active regions on the Martian surface. If viewed as a byproduct of methane oxidation, the presence of formaldehyde would indicate that much more methane was being produced each year than previously thought - pointing towards a current and continuous source of methane production [6-8].

Formaldehyde formation at altitudes above $80 \mathrm{~km}$ is dominated by the photodissociation of methane followed by reaction with carbon dioxide $\left(\mathrm{CO}_{2}\right)$ to form formaldehyde [8].

$$
\mathrm{CH}_{4} \stackrel{h v}{\longrightarrow}{ }^{3} \mathrm{CH}_{2} \stackrel{\mathrm{CO}_{2}}{\longrightarrow} \mathrm{CH}_{2} \mathrm{O}
$$




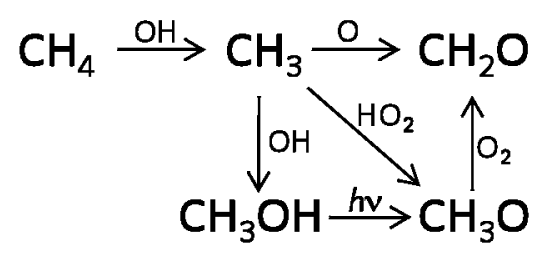

At altitudes below $80 \mathrm{~km}$, methane is oxidized by $\mathrm{OH}$ to form $\mathrm{CH}_{3}$, which then reacts with either $\mathrm{O}$ to form formaldehyde directly or via reactions with $\mathrm{OH}$ or $\mathrm{HO}_{2}[8]$.

Wong et al. [8] found the modeled production of hydrocarbons through methane photochemistry to be very low even for high methane abundances in the atmosphere. In the upper atmosphere, they attribute this to the competing photolysis between methane and carbon dioxide - which have overlapping cross sections. In the lower atmosphere, oxidation of methane by $\mathrm{OH}$ competes with the removal of $\mathrm{OH}$ by $\mathrm{CO}$ and $\mathrm{O}$.

There is some variation in the modeled lifetimes of methane and formaldehyde in the Martian atmosphere. In 2002, Summers et al. [7] reported a methane lifetime of $\sim 300$ years and a formaldehyde lifetime of $\sim 2$ weeks. In 2004, Wong et al. [8] gave photochemical lifetimes for $\mathrm{CH}_{4}$ and $\mathrm{CH}_{2} \mathrm{O}$ of 670 years and 7.5 hours respectively. In 2009, Mumma et al. [9] compared observed measurements of methane in spring 2006 with the amount of methane released in a plume observed in March 2003 and found the 2006 mixing ratios to contradict with the estimated lifetime for methane. They concluded that a process other than photochemical destruction must be responsible for the majority of the methane removal and found the destruction lifetime of methane to range from $\sim 0.6$ to $\sim 4$ Earth years depending on whether the large release was a onetime, or annually occurring event.

Measurements of the formaldehyde abundance in the Martian atmosphere have been reported by two sources. In 1993, Korbalev et al. [10] observed formaldehyde during the Phobos mission through solar occultation observations in the equatorial spring. They tentatively reported an average mixing ratio of $\sim 0.5 \mathrm{ppm}$. In 1997, Krasnopolsky et al. [11] measured significantly less formaldehyde $(\sim 3 \mathrm{ppb})$ through a combination of 4 meter telescope at the Kitt Peak National 
Observatory (KPNO) and Fourier transform spectrometer with Goddard Space Flight Center (GSFC) postdisperser. For the purposes of instrument design, we use the more conservative mixing ratio and assume that a detection limit of $\sim 1 \mathrm{ppb}$ is necessary.

Gas correlation radiometry is a mature sensing technology on Earth, and with successful miniaturization, it holds great promise for trace gas measurements in the Martian atmosphere. An instrument developed for a Mars orbit will likely have greater restrictions on mass and volume than an Earth orbiter. The component that most impacts the size of a gas correlation radiometer is the gas correlation cell - the pathlength of which affects the sensitivity of the instrument. MOPITT (Measurements of Pollution in the Troposphere) is a satellite gas correlation radiometer for measuring carbon monoxide (CO) and methane in the Earth's atmosphere [12-14]. They use two techniques to improve sensitivity while limiting the overall length of the gas correlation cell - a pressure modulation technique which changes the gas cell pressure using a piston, and a length modulation technique which changes the gas pathlength with a rotating disk of calcium fluoride in the vacuum cell. These approaches significantly impact the complexity level, and mass and power requirements of the instrument. Tolton[15] presented a strawman concept for an Earth-orbiting gas correlation radiometer for measuring $\mathrm{CO}_{2}$. His design implemented a multipass gas correlation cell to reduce the instrument footprint. Here we present preliminary work on a miniaturized gas correlation radiometer for measuring formaldehyde that is less complex than the MOPITT approach, with significantly smaller mass and volume than the Tolton design.

\section{Instrument design}

\subsection{Gas correlation radiometer}

A schematic of the current instrument configuration is shown in figure 1. To simulate the effect of light passing through the atmosphere, light from a silicon carbide (SiC) lamp (2-10 $\mu \mathrm{m})$ passes through a 2 meter absorption cell (Axiom Analytical, part number LFT-220A with 
sapphire windows) in the test set-up (shown outside the dashed line). The absorption cell was filled with a range of gas mixing ratios $(0-42 \mathrm{ppm}$ formaldehyde in nitrogen) to demonstrate instrument sensitivity. The gas correlation radiometer instrument is shown within the dashed region of figure 1. Incoming light that has undergone absorption by trace gas is focused, modulated with an optical chopper, and re-collimated. Light then passes through a narrow bandpass filter at a wavelength that selects absorption features of the trace gas while minimizing interference from other spectral features. In the current set-up, the bandpass filter (Spectrogon) is centered at $3.62 \mu \mathrm{m}$ with a $40 \mathrm{~nm}$ bandwidth. After passing through the narrow bandpass filter, light is split into two channels with a pellicle beamsplitter (Thorlabs, CM1-BP4).

Off-axis parabolic (OAP) mirrors launch light from these channels into the hollow waveguides through modified vacuum fittings (discussed in Section 2.2) that also serve as waveguide terminators. In the first channel, a sample of formaldehyde (1 atm of $31.8 \mathrm{ppm} \mathrm{H}_{2} \mathrm{CO}$ in a balance of $\mathrm{N}_{2}$ ) is enclosed in a $1 \mathrm{~mm}$ inner diameter, 3 meter long hollow waveguide and is used as a spectral filter - effectively blocking atmospheric absorption by this gas so that this

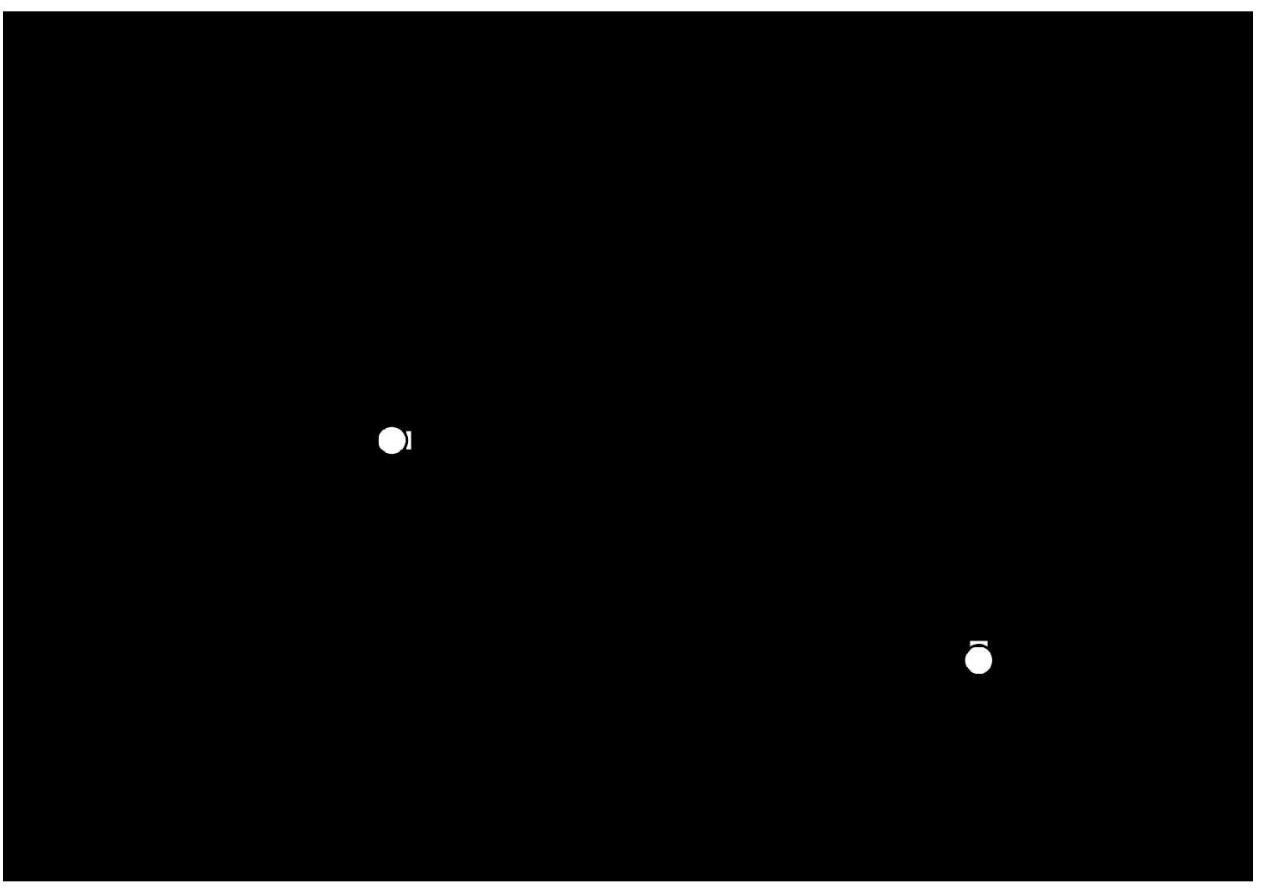

Figure 1. Instrument schematic. Hollow waveguides replace conventional gas-correlation radiometer absorption cells - resulting in significant mass and volume savings. 
channel is sensitive to changes in solar flux but not to changes in the trace gas. The second channel has an identical, but evacuated hollow waveguide that is sensitive to changes in atmospheric absorption by the trace gas (this channel is more sensitive to changes in absorption than changes in flux).

The ratio of channel 2 to channel 1 is sensitive to changes in absorption but not to changes in solar flux. Use of duplicate hollow waveguides (filled and evacuated) reduces etalon effects in the ratio. Light exiting from the waveguides is measured with thermo-electrically cooled $\mathrm{HgCdTe}$ detectors (Judson, J19TE). Signals are processed through analog lock-in amplifiers (Stanford Research Systems, SR830) referenced to the frequency of the chopped signals. Data is handled through Signal Express (Labview) software.

\subsection{Hollow waveguide}

The hollow silica waveguides used for gas correlation absorption cells are manufactured by Polymicro Technologies. These waveguides are comprised of silica tubing, with a protective acrylate coating. Within the silica tubing is a silver film layer, and finally a silver iodide layer at the interior of the waveguide. This waveguide propagates light in the IR between approximately $2.9 \mu \mathrm{m}$ and $10.6 \mu \mathrm{m}$. At $3.6 \mu \mathrm{m}$ wavelength the manufacturer reports a spectral attenuation of less than one $\mathrm{dB} / \mathrm{m}$. Manufacturer tests of the straight and bend loses of this waveguide are reported not to exceed $1.0 \mathrm{~dB} / \mathrm{m}$ and $1.5 \mathrm{~dB} / \mathrm{m}$. The mass of the waveguide is $4.64 \mathrm{~g} / \mathrm{m}$.

To maximize the amount of light that could be used by the instrument, we chose the largest available waveguide diameter (1000 $\mu \mathrm{m}$ I.D., model HWEA10001600). The aperture for the entire system is limited by the aperture with the smallest etendue. We assume that the waveguide will be the limiting aperture in this system. The etendue is the product of the area of an aperture times the solid angle that the aperture can accept. The area of aperture is given as

$$
\mathrm{A}=\pi\left(\frac{f}{2 N}\right)^{2}, N=\frac{f}{D}
$$


where $f$ is the focal length and $N$ is the f-number, and $\mathrm{D}$ is the diameter of the entrance pupil. We estimated the waveguide's acceptance angle to be $\sim 8^{\circ}$ by launching SiC lamp light through a 0.1 $\mathrm{m}$ segment of the waveguide and measuring the exit divergence angle. For a waveguide with a core diameter of $1000 \mu \mathrm{m}$, and an acceptance angle of $\sim 8^{\circ}$, the etendue would be

$$
G=\frac{\pi}{4}(0.1)^{2} \cdot\left(\frac{\pi}{180} \cdot 8\right)^{2} \cdot \pi
$$

in units of $\mathrm{cm}^{2}$. Placing a large telescope at the entrance to the instrument cannot increase the amount of light gathered to make measurements. The only effect of the telescope is to control the size of the instrument's footprint on the ground.

Waveguide terminations are not currently available from the manufacturer for this diameter. However, we were able to modify $1 / 16$ " Swagelok union cross tube fittings (SS-100-4) with a central bore and a sapphire window (Judson Technologies) to provide a stable fitting for the waveguide that does not interfere with light launching, and doubles as a vacuum connection for gas addition.

\subsection{Simulations}

The performance of a Mars orbiting version of the hollow-core fiber instrument was simulated assuming a 3 meter long, 1000 micron inner diameter hollow-core fiber gas correlation cell, a 92.8 degree sun-synchronous orbit from $400 \mathrm{~km}$ with a horizontal sampling scale of $10 \mathrm{~km}$ x $10 \mathrm{~km}$. The simulation generates a synthetic spectrum for reflected light containing spectral features from the HITRAN[16] database. It should be noted that the HITRAN database is intended for evaluating absorption in the Earth's atmosphere and consequently, some bands found in the Martian atmosphere have been neglected or are incomplete in this database. However, it is still useful for some initial performance simulations - as long as final spectral selections are not based on this alone. 
The reflected solar flux signal received in the two channels of each sub-instrument (evacuated and gas-filled correlation cells) is then calculated for light that has passed through the Martian atmosphere, reflected off the surface, and passed back up to the spacecraft. For each simulation, the total column of the species of interest is perturbed by $1 \%$ and the signals are recalculated. We compare the change in the ratio (filled cell to evacuated cell) caused by the perturbation to the detector noise of this ratio. The free parameters of the instrument design (filter bandpass, filter edge slopes, instrument FOV, etc.) are varied in an effort to maximize the response to the species measured. Initial results indicate that for one second of averaging $(3 \mathrm{~km}$ displacement along the satellite ground track), a detection limit of slightly better than $1 \mathrm{ppb}$ is possible for formaldehyde.

For these experiments, purchase of a prefilter with optimal parameters (based on these simulations) was cost prohibitive. Instead, we purchased a number of off-the-shelf bandpass filters in the $3.6 \mu \mathrm{m}$ region and modeled their performance with these simulations. Figure 2

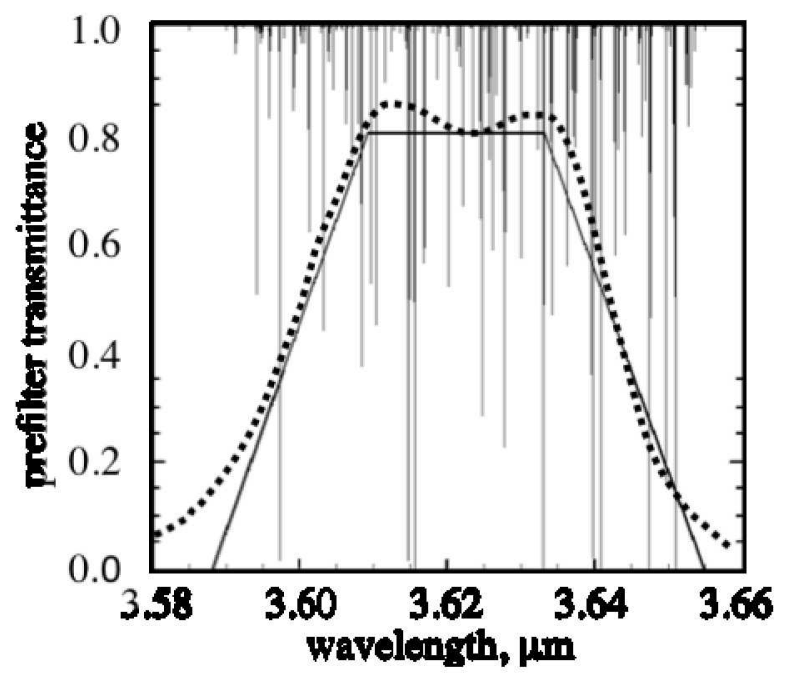

Figure 2. The bandpass of the prefilter (dashed line) used in these experiments is shown overlaid on formaldehyde absorption features. The simulated bandpass of this prefilter is shown as a solid line.

illustrates the absorption features selected by the most optimal of these filters. An FTIR scan of the prefilter selected for these experiments (dashed line) is shown with the simulated prefilter 
bandpass (solid line) overlaid on formaldehyde absorption features in $\mathrm{P}$ branch of the $\mathrm{a}_{1}$ band centered at $3.594 \mu \mathrm{m}\left(2782 \mathrm{~cm}^{-1}\right)$. The selected bandpass has formaldehyde absorption features as well as interfering features (methane, water vapor, etc.). Selection of a more optimal bandpass filter will minimize contributions from these interfering absorption features. However to achieve the required selectivity in the final instrument design, sample and reference waveguides will likely contain mixtures of known interfering gases - resulting in an instrument that is not sensitive to these interfering gases. For example, if the waveguide in channel 1 is filled with formaldehyde and methane, and the waveguide in channel 2 is filled with methane, the ratio of channel 2 to channel 1 would be sensitive to changes in formaldehyde but not to changes in methane.

\section{Results}

\subsection{Formaldehyde measurements}

In these measurements, the channel 1 waveguide was filled with $31.8 \mathrm{ppm}$ formaldehyde in a balance of nitrogen for a total waveguide pressure of 1 atm (760 torr). Maintaining the waveguide pressure at atmospheric pressure reduced the likelihood of long-term contamination of air into the waveguide. To track the sensitivity of the instrument to changes in the formaldehyde concentration, the 2 meter absorption cell (located at the front end of the instrument) was filled with concentrations ranging from 0 to $41.8 \mathrm{ppm}$ formaldehyde in nitrogen while monitoring changes in the ratio of channel 2 to channel 1. Signals from both channels were recorded for an integration time of 1 second. Representative data from these experiments is shown in figure 3. The linear regression of this data is shown as a solid line, and the square of the correlation coefficient is 0.995 . Vertical error bars indicate the standard deviation for each data point. 


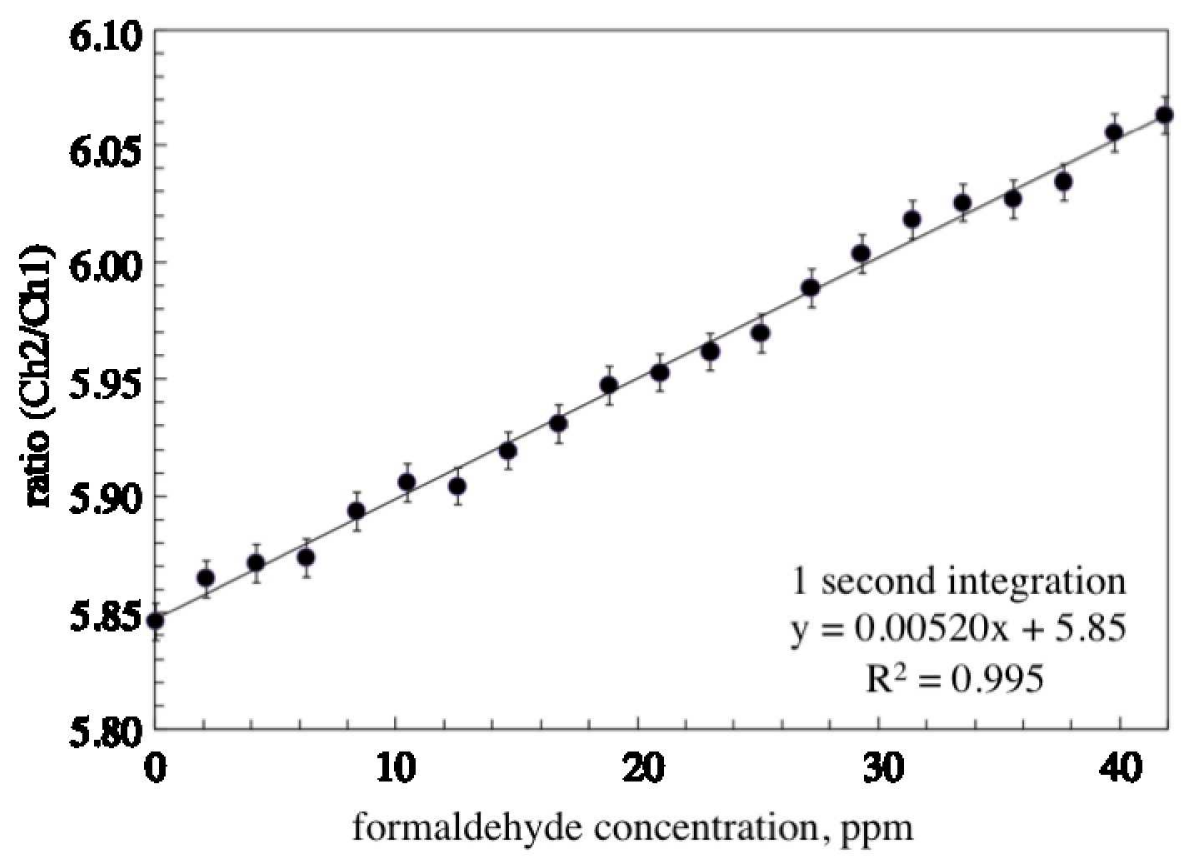

Figure 3. Instrument sensitivity to changes in the formaldehyde concentration in a 2 meter infrared gas cell. The integration time for each data point is one second. Solid circles represent an average of three data points measured at each concentration, with vertical error bars indicating the standard deviation of the three measurements. In this series, the ratio changes by 0.00520 per ppm change in formaldehyde concentration. This is approximately equivalent to a $30 \mathrm{ppb}$ sensitivity to formaldehyde in the Martian atmosphere.

A ratio change of 0.217 can be seen over this concentration range, resulting in a slope of 0.00520 which represents the change in ratio per ppm change in formaldehyde. Assuming a signal to noise ratio of $2: 1$, we estimate that in the lab the instrument is sensitive to formaldehyde changes of 3 ppm or greater. We related the sensitivity of the instrument to changes in formaldehyde concentration in the 2 meter cell to expected changes in the Martian atmosphere through the Bouguer-Lambert Law where absorbance is given as

$$
-\ln \left(\frac{I}{I_{o}}\right)=S \rho g \chi \ell
$$

where $S$ is the linestrength from HITRAN, $\rho$ is the density, $g$ is the linewidth, $\chi$ is the mole fraction, and $\ell$ is the pathlength. 
In the 2 meter cell, the temperature was constant at $293 \mathrm{~K}$ and we assumed an average temperature in the Martian atmosphere of $210 \mathrm{~K}$. The cell pressure was 1 atm and the average atmospheric pressure on Mars was estimated to be $6.91 \times 10^{-3} \mathrm{~atm}$. We assumed $S$ to be approximately constant in both environments; linestrength values of formaldehyde at $210 \mathrm{~K}$ and 293K in HITRAN were found to be equivalent to four decimal places over the bandpass wavelength range. In both environments, formaldehyde lines are assumed to have a Voight lineshape, with a greater lorenzian contribution in the lab due to the higher pressure and temperature in the 2 meter cell relative to that in the Martian atmosphere. The density in both environments is found through the ideal gas law. We assume that with an orbital instrument, sunlight would pass through $11.1 \mathrm{~km}$ atmosphere twice (once from the sun to the Martian surface, and then once from the surface to the satellite) for a total pathlength of $22.2 \mathrm{~km}$. With these assumptions, the absorbance difference was calculated for a $3 \mathrm{ppm}$ change in the formaldehyde mole fraction in the absorption cell. This was found to be approximately equivalent to a $30 \mathrm{ppb}$ change in formaldehyde in the Martian atmosphere.

In figure 4, a representative segment of the instrument noise is shown for both $1 \mathrm{~ms}$ and 1 $\mathrm{s}$ averages with an enlargement of the $1 \mathrm{~s}$ average shown inset. The one second average time is significant because it represents a $3 \mathrm{~km}$ displacement along the satellite ground track. The standard deviation of the ratio in the noise data with 1 second integration is 0.00425 . If we assume a 3:1 signal to noise ratio is required for the detection limit, this would indicate that only changes in the ratio greater than 0.0128 are detectable. The ratio in figure 3 changes by 0.00520 per ppm increase in formaldehyde concentration. This approximates the current instrument detection limit to be slightly less than $3 \mathrm{ppm}$ in the lab. To estimate the performance of the instrument in a Mars orbit, we assume an average atmospheric pressure on Mars of $\sim 7$ millibars, two passes through the atmosphere at $\sim 22.2 \mathrm{~km}$, and a temperature of $210 \mathrm{~K}$. With these scaling 


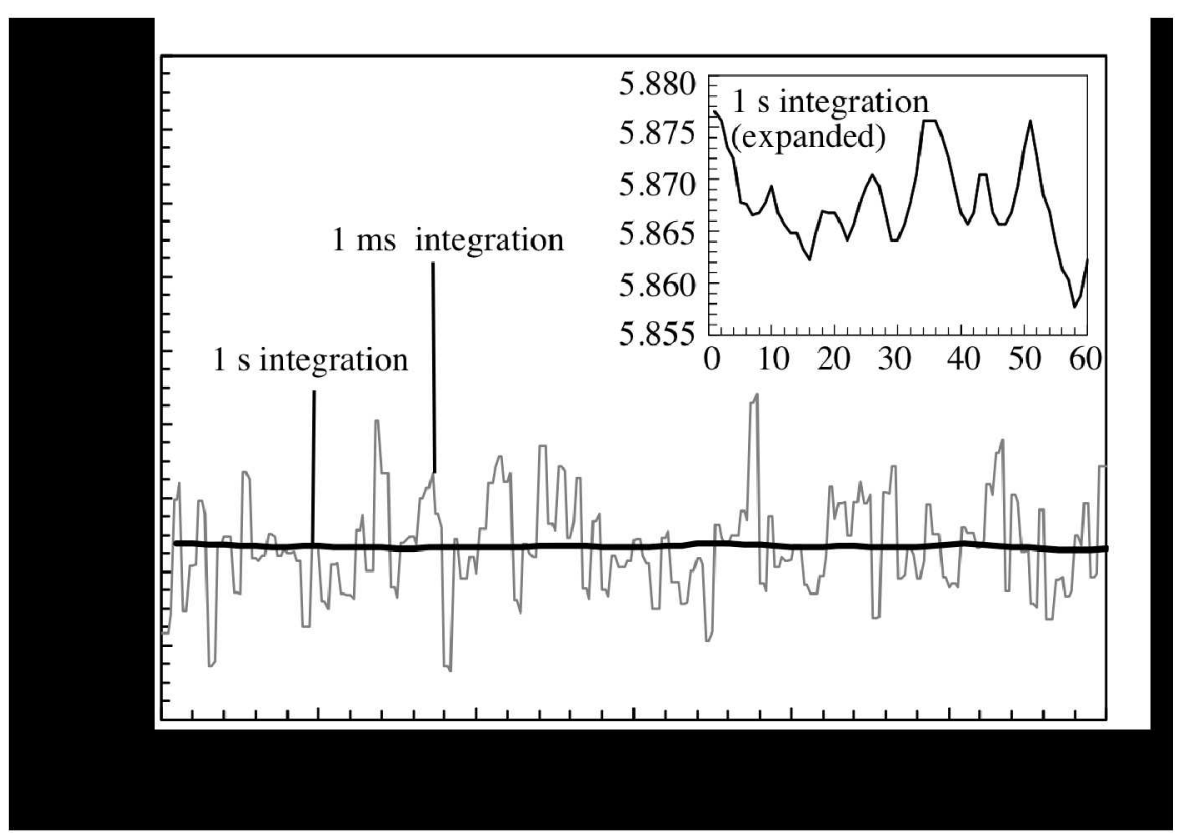

Figure 4. A representative segment of instrument noise is compared at $1 \mathrm{~ms}$ and $1 \mathrm{~s}$ integration times. An enlargement of the 1 second noise is shown inset. The noise limited detection limit is slightly less than $3 \mathrm{ppm}$ for the lab instrument.

factors, it is estimated that the instrument in this preliminary configuration would have a detection limit of approximately $30 \mathrm{ppb}$ in the Martian atmosphere.

\section{Limitations and Opportunities}

The goal of this effort was to demonstrate that miniaturization of a gas correlation radiometer was possible by implementing a hollow waveguide as a gas correlation cell. While this proof-of principle was successful, for this instrument to be viable for measurements of formaldehyde on Mars, there would have to be improvements in both the detection limit and trace gas selectivity.

For localizing sources of formaldehyde in the Martian atmosphere, a detection limit of approximately $1 \mathrm{ppb}$ is required. In the current configuration, the instrument is estimated to have a detection limit of approximately $30 \mathrm{ppb}$ in the Martian atmosphere. We anticipate that an 
optimized optical design as well as future improvements in thermal control and structural stability of the instrument will improve this value.

The wavelength region selected by the bandpass filter is key to selecting formaldehyde absorption features while limiting interfering absorption features such as methane and water vapor as well as interferences due to Fraunhofer lines and planetary thermal emission. The simulations discussed in section 2.3 were used to select the most advantageous spectral region. However, a prefilter with these parameters was cost prohibitive for this early level of development. A future more optimal filter will offer significant improvement to the instrument selectivity. In addition to prefilter selection, selectivity will also be improved by filling both waveguides with mixtures of interfering gases as discussed in section 2.3 to remove sensitivity to these gases.

The relative simplicity of this technique provides an opportunity for the instrument to be expanded to measure a range of atmospheric trace gases of interest on Mars such as methane, water vapour, deuterated water vapour, nitrous oxide, hydrogen sulfide, methanol, sulfur dioxide, and ethane. Methane and water vapour instruments are the focus of parallel efforts.

\section{Conclusions}

We have presented progress on the development of a low-cost miniaturized gas correlation radiometer that implements a hollow waveguide as a gas correlation cell. The substantial reduction in mass and volume of the gas correlation cell makes this technology appropriate for an orbital or aircraft probe mission - capable of pinpointing sources of trace gases in the Martian atmosphere. We have demonstrated this technique as a fomaldehyde sensor and report a detection limit of slightly better than $3 \mathrm{ppm}$ in the lab, and an estimated detection limit of $\sim 30 \mathrm{ppb}$ in a Martian orbit. Simulated performance of an optimized Mars orbiting formaldehyde instrument indicates that a detection limit of $1 \mathrm{ppb}$ is possible, however future improvements in both sensitivity and selectivity of the current prototype are necessary to reach this target. 
Significant differences between the composition of the Mars and Earth atmospheres indicate a need for more careful spectroscopic analysis to account for potential interfering absorption features as well as Fraunhofer lines and planetary thermal emission.

\section{Acknowledgments}

This research was supported by the NASA Planetary Instrument Definition and Development Program and the NASA Goddard Space Flight Center Internal Research and Development program. 


\section{References}

[1] Farmer B 2006 MEPAG MARS SCIENCE ORBITER (MSO) MSO Science Analysis Group 1-48

[2] Formisano V, Atreya S, Encrenaz T, Ignatiev N, Giuranna M 2004 Detection of Methane in the Atmosphere of Mars Science 3065702 1758-61

[3] Onstott TC, McGown D, Kessler J, Sherwood B, Lehmann K, Clifford SM 2006 Martian CH4: Sources, Flux, and Detection Astrobiology 62 377-95

[4] Hinrichs K-U, Hayes JM, Bach W, Spivack AJ, Hmelo LR, Holm NG, et al. 2006 Biological formation of ethane and propane in the deep marine subsurface Proc Natl Acad Sci $1034014684-89$

[5] Wanke H, Dreibus G 1992 The role of SO2 on Mars American Astronomical Society, 24th DPS Meeting 2426.10987

[6] Summers ME, editor. Fate of Disequilibrium Trace Gases in the Martian Atmosphere. Methane on Mars: NASA Astrobiology Institute (NAI) Distributed Workshop; 2005: NASA Goddard Space Flight Center.

[7] Summers ME, Lieb BJ, Chapman E, Yung YL 2002 Atmospheric biomarkers of subsurface life on Mars Geophysical Research Letters 2924 2171-74

[8] Wong AS, Atreya SK, Formisano V, Encrenaz T, Ignatiev NI 2004 Atmospheric photochemistry above possible martian hot spots Advances in Space Research 33 2236-39

[9] Mumma MJ, Villanueva GL, Novak RE, Hewagama T, Bonev BP, DiSanti MA, et al. 2009 Strong Release of Methane on Mars in Northern Summer 2003 Science 323 1041-45

[10] Korablev O, Ackerman M, Krasnopolsky VA, Moroz V, Muller C, Rodin C, et al. 1993 Tentative identification of formaldehyde in the Martian atmosphere Planetary Space Science 416 441-51

[11] Krasnopolsky VA, Bjoracker GL, Mumma MJ, Jennings DE 1997 High resolution Spectroscopy of Mars at 3.7 and $8 \mathrm{~m}$ : A Sensitive Search for $\mathrm{H} 2 \mathrm{O} 2, \mathrm{H} 2 \mathrm{CO}, \mathrm{HCl}$, and CH4, and Detection of HDO J Geophys Res 102 E3 6525-43

[12] Liu J, Drummond JR, Li Q, Gille JC, Ziskin DC 2005 Satellite mapping of CO emission from forest fires in Northwest America using MOPIITT measurements Remote Sensing of Environment 95 502-16

[13] Drummond JR, Bailey PL, Brasseur G, Davis GR, Gille JC, Peskett GD, et al. 1999 Early Mission Planning for the MOPITT Instrument wwwatmospphysicsutorontoca/MOPITT/missionpdf

[14] Megonigal JP, Guenther AB 2008 Methane emissions from upland forest soils and vegetation Tree Physiology 28 491-98

[15] Tolton BT, Hackett J, Caldwell D, Miller D 2004 Strawman design for a gas-filter correlation radiometer satellite instrument to measure the atmospheric $\mathrm{CO} 2$ column Proc of SPIE 5543 332-37

[16] Rothman LS, Jacquemart D, Barbe A, Benner DC, Birk M, Brown LR, et al. 2005 The HITRAN 2004 molecular spectroscopic database $J$ Quant Spectrosc Radiat Transf 962 139-204 